\title{
Specimen Type Code
}

National Cancer Institute

\section{Source}

National Cancer Institute. Specimen Type Code. NCI Thesaurus. Code C95396.

A coded value specifying the kind of specimen. 